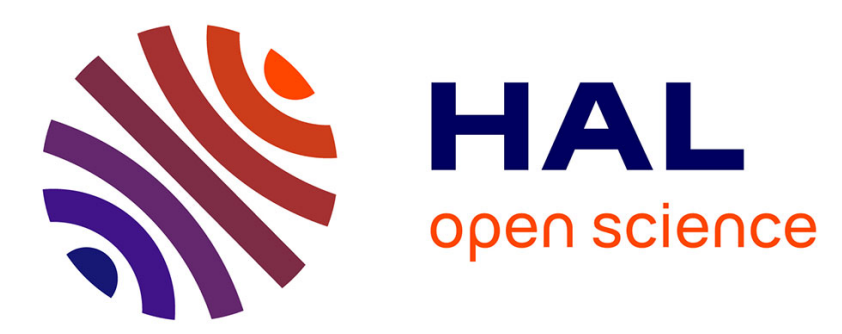

\title{
A Risk-Based Sensor Management using Random Finite Sets and POMDP
}

Marcos Eduardo Gomes Borges, Dominique Maltese, Philippe Vanheeghe, Emmanuel Duflos

\section{- To cite this version:}

Marcos Eduardo Gomes Borges, Dominique Maltese, Philippe Vanheeghe, Emmanuel Duflos. A RiskBased Sensor Management using Random Finite Sets and POMDP. 20th International Conference on Information Fusion (FUSION), Jul 2017, Xi'an, China. pp.1-9, 10.23919/ICIF.2017.8009843 . hal-02411689

\section{HAL Id: hal-02411689 \\ https://hal.science/hal-02411689}

Submitted on 15 Dec 2019

HAL is a multi-disciplinary open access archive for the deposit and dissemination of scientific research documents, whether they are published or not. The documents may come from teaching and research institutions in France or abroad, or from public or private research centers.
L'archive ouverte pluridisciplinaire HAL, est destinée au dépôt et à la diffusion de documents scientifiques de niveau recherche, publiés ou non, émanant des établissements d'enseignement et de recherche français ou étrangers, des laboratoires publics ou privés. 


\title{
A Risk-Based Sensor Management using Random Finite Sets and POMDP
}

\author{
Marcos Eduardo Gomes-Borges*†, Dominique Maltese ${ }^{\dagger}$, Philippe Vanheeghe*, Emmanuel Duflos* \\ ${ }^{*}$ Centrale Lille, CRIStAL, UMR 9189, 59650 Villeneuve d'Ascq, France \\ ${ }^{\dagger}$ Safran Electronics \& Defense, 91344 Massy, France
}

\{marcos.borges, dominique.maltese\}@ safrangroup.com

\{philippe.vanheeghe, emmanuel.duflos\}@ centralelille.fr

\begin{abstract}
In this paper, we consider the problem of scheduling an agile sensor to perform an optimal control action in the case of the multi-target tracking scenario. Our purpose is to present a random finite set (RFS) approach to the multitarget sensor management problem formulated in the Partially Observed Markov Decision Process (POMDP) framework. The reward function associated with each sensor control (action) is computed via the Expected Risk Reduction between the multitarget predicted and updated densities. The proposed algorithm is implemented via the Probability Hypothesis Density filter (PHD). Numerical studies demonstrate the performance of this particular approach to a radar beam-pointing problem where targets need to be prioritized.
\end{abstract}

\section{INTRODUCTION}

Advances in sensor technologies led to the emergence of a large number of controllable degrees of freedom in sensing devices. Sensor management becomes relevant when the sensing system has the capability of actively managing these resources in reaction to previous measurements [1], [2]. Examples of systems in which sensor management is currently used include autonomous robots, unmanned aerial vehicles, surveillance, waveform-agile radars, and pan-tilt-zoom cameras. The term Sensor Management refers to the control of the degrees of freedom in an agile sensor system to satisfy operational constraints and achieve operational objectives [1]. This paper focuses on the problem of scheduling a multifunction radar (MFR) to perform an optimal control action in order to track an unknown number of targets.

The multi-target tracking is one of the main applications of sensor management. It refers to the problem of jointly estimating the number of targets and their states or trajectories from noisy sensor measurements [3]-[5]. The key to a successful multi-target tracking lies in the optimal extraction of useful information about the target's state from the observations in the presence of sensor's imperfections. This task is usually realized by maintaining an estimate of the target's state over time using algorithms such as the Multiple Hypothesis Tracking (MHT) and the Join Probabilistic Data Association (JPDA) [4]. The main characteristic of these approaches is the strategy applied to solve the data association problem which unfortunately results in a non-Bayes-optimal estimation [5].
More recently, an alternative formulation that avoids explicit associations between measurements and targets was proposed by Mahler [6]. This new approach implements the Random Finite Set (RFS) theory in order to solve the multi-target tracking problem in a Bayesian framework. It offers a mathematically elegant and intuitive representation of a finite but time-varying number of targets and measurements [5], [6]. The optimal multi-target Bayes filter based on RFS is computationally intractable due to the fact that, in general, it involves multiple integrals that have no closed form solutions [6], [7]. To overcome these limitations, approximations are required. Several algorithms, such as the Probability Hypothesis Density (PHD) filter [6], the Cardinalized PHD (CPHD) filter [8], the Multi-Object Multi-Bernoulli (MeMBer) filter [9], [10], and the Generalized Labeled Multi-Bernoulli (GLMB) filter [11], [12], were proposed.

Multi-target sensor management is typically an optimal nonlinear control problem. Its objective is to allocate resources optimally directing the right sensor on the right platform to the appropriate target at the proper time [1], [13]. Also, in the case of the multi-target sensor management problem, not only does the number of targets vary in time, but also the measurements are susceptible to miss detections and false alarms. With reference to the RFS approach, Mahler developed theoretical foundations of the multi-target sensor management reward function relating to the posterior expected number of targets (PENT) [14]. In order to identify targets of importance, the author also proposed the posterior expected number of targets of interest (PENTI) tasking reward function [5], [15]. Delande, in turn, introduced a reward function called Balanced Explorer and Tracker (BET) that provides an efficient sensor management in situations where the sensor's field of view cannot cover the whole state space at the same time [16]. Ristic and Vo proposed a reward function to sensor management using the Rényi divergence between the multi-target prior and multi-target posterior densities [17], [18]. More recently, Hoang, Vo, Vo, and Mahler offered a new intuitive and tractable objective function based on the Cauchy-Schwartz information functional [19].

When only a subset of the total targets can be successfully tracked, the prioritization of target tracks is crucial and cannot 
be achieved by means of information gain-based metrics. To overcome the limitations of existing metrics, a statistical risk model used to calculate an expected cost as a metric, started to be applied in recent research. Papageorgiou et. al. proposed a risk-based approach to sensor resource management for the problem of missile defense [20]. Wang et. al. developed a Bayesian risk sensor management for integrated detection and estimation [21]. Martin introduced a statistical risk-based metric for a field of view problem [22]. The current research differs from the above-mentioned studies in that it presents a risk-based sensor management using the RFS and the POMDP framework.

This paper is devoted to sensor management using the PHD filter and can be seen as a sequel to [23] in which a kinematic state estimation based on the Extended Kalman filter was carried out. We define the Expected Risk Reduction (ERR) approach to multi-target sensor management which is similar to that in [20]-[23]; however, to jointly estimate the number of targets and their states, the GM-PHD tracker [24], [25] is implemented.

We take into consideration a surveillance context with electromagnetic emission constraints as presented in [26]. It is assumed that there are too many maneuverable targets to be tracked by the radar system. Only a subset of all the targets need to be tracked and, initially, we have knowledge of their kinematic states, yet their classification states are unknown. Finally, it is also presumed that there is a cost resulting from an incorrect decision on a target's true classification. Thus, the task of a sensor manager is to decide which targets the sensor should focus on in order to reduce the expected cost of an incorrect classification decision.

Section II presents a brief background on Finite Set Statistics (FISST) and the PHD filter. Section III describes the derivation and implementation of the expected risk reduction as a reward function for PHD filter with controllable sensors. Section IV demonstrates the performance of the proposed reward function in the context of multi-target tracking in a radar beam-pointing problem where targets need to be prioritized. The conclusions are presented in Section V.

\section{Finite Set Statistics AND The PHD Filter}

In this paper we consider that the task of a sensor manager is not to reduce the uncertainty in the target state estimation, nor to make classification decisions on target tracks. Instead, its role revolves around actively scheduling and managing sensor resources to chose the best control action that will result in the most significant reduction in the expected cost of making an incorrect classification decision. The cost associated with different sensor actions is computed using the prior and posterior multi-target densities. The calculation of these densities requires a Bayesian multi-target filter, which is described hereafter.

In a multi-target tracking, random finite sets are used to model uncertainty in the multi-object state $X_{k} \subset \mathbb{X}$ and multi-object measurement $Z_{k} \subset \mathbb{Z}$ at each time $k$. Due to imperfections of detectors, it is possible that at time $k$ some of the targets in $X_{k}$ are not detected. Furthermore, the observation set $Z_{k}$ normally includes false detections and clutter in addition to target-originated detections [5].

The objective of the recursive Bayesian multi-object statespace estimator is to determine, at each time $k$, the posterior probability density of multi-object state $\pi_{k}\left(X_{k} \mid Z_{1: k}\right)$, where $Z_{1: k}=\left(Z_{1}, \cdots, Z_{k}\right)$ denotes the observation set sequence up to time $k$. The multi-object posterior density can be computed recursively via the prediction and update steps. The predicted density at time $k$, denoted as $\pi_{k \mid k-1}\left(X_{k} \mid Z_{1: k-1}\right)$, is computed by the multi-object Chapman-Kolmogorov equation [5]:

$\pi_{k \mid k-1}\left(X_{k} \mid Z_{1: k-1}\right)=\int f_{k \mid k-1}\left(X_{k} \mid X\right) \pi_{k-1}\left(X_{k} \mid Z_{1: k-1}\right) \delta X$

where $f_{k \mid k-1}\left(X_{k} \mid X\right)$ is the Markov transition density from time $k-1$ to time $k$. When new observations are available, which is described by a multi-object likelihood function $g_{k}\left(Z_{k} \mid X_{k}\right)$, the new posterior density is computed via the multi-object Bayes rule:

$$
\pi_{k}\left(X_{k} \mid Z_{1: k}\right)=\frac{g_{k}\left(Z_{k} \mid X_{k}\right) \pi_{k \mid k-1}\left(X_{k} \mid Z_{1: k-1}\right)}{\int g_{k}\left(Z_{k} \mid X\right) \pi_{k \mid k-1}\left(X \mid Z_{1: k-1}\right) \delta X}
$$

\section{A. The PHD filter}

In general, computing the exact Bayesian multi-object posterior is intractable, and approximations are required. In this paper, the PHD filter is treated as an approximation to the multi-object Bayesian filter to propagate the multi-object posterior density recursively in time. Given the posterior intensity $D_{k-1}$ at time $k-1$, the intensity function $D_{k \mid k-1}$ to time $k$, the PHD recursion computes the posterior intensity function of the target RFS as follows:

$$
\begin{aligned}
D_{k \mid k-1}(x)=\gamma_{k}(x)+\int & \left(p_{S, k}(\xi) f_{k \mid k-1}(x \mid \xi)\right. \\
+ & \left.\beta_{k \mid k-1}(x \mid \xi)\right) D_{k-1}(\xi) d \xi
\end{aligned}
$$

$$
\begin{aligned}
& D_{k \mid k}(x)=\left[1-p_{D, k}(x)\right] D_{k \mid k-1}(x) \\
& \quad+\sum_{z \in Z_{k}} \frac{p_{D, k}(x) g_{k}(z \mid x) D_{k \mid k-1}(x)}{\kappa_{k}(z)+\int p_{D, k}(\xi) g_{k}(z \mid \xi) D_{k \mid k-1}(\xi) d \xi}
\end{aligned}
$$

where $\gamma_{k}(\cdot)$ denotes the intensity of spontaneous target birth at time $k ; \beta_{k \mid k-1}(\cdot \mid \xi)$ represents the intensity of the target RFS spawned by a target of previous state $\xi$ at time $k ; p_{S, k}(\xi)$ is the probability that a target still exists at time $k$ given that its previous state is $\xi ; p_{D, k}(x)$ stands for the probability of detections given a state $x$ at time $k ; \kappa_{k}(\cdot)$ is the intensity of the clutter RFS and equals $\gamma_{c} C_{k}(\cdot)$ (assumed to be Poisson).

In general, the PHD recursion given in (3)-(4) do not admit closed-form solutions. Under linear Gaussian assumptions, the multi-object posterior density can be approximated using the GM-PHD filter. In the remainder of this section we summarize 
the main points of the GM-PHD filter, and for a complete description, see [27], [28].

\section{B. Linear Gaussian Multi-Target Models}

The linear Gaussian multi-target model includes certain assumptions on the birth, death and detection of targets. They are summarized as follows:

1) Each target follows a linear Gaussian dynamical model, i.e.,

$$
\begin{aligned}
f_{x \mid k-1}(x \mid \zeta) & =\mathcal{N}\left(x ; F_{k-1} \zeta, Q_{k-1}\right) \\
g_{k}(z \mid k) & =\mathcal{N}\left(z ; H_{k} x, R_{k}\right)
\end{aligned}
$$

where $F_{k-1}$ is the state transition matrix and $Q_{k-1}$ is the process noise covariance, $H_{k}$ is the observation matrix, and $R_{k}$ is the observation noise covariance. The term $\mathcal{N}(\cdot ; m, P)$ is the Gaussian density with mean $m$ and covariance $P$.

2) The detection and survival probabilities are both state independent, i.e., $p_{D, k}(x)=p_{D, k}$ and $p_{S, k}(x)=p_{S, k}$.

3) The intensities of the birth and spawn RFSs are both Gaussian mixtures of the form:

$$
\begin{aligned}
\gamma_{k}(x)= & \sum_{i=1}^{J_{\gamma, k}} w_{\gamma, k}^{(i)} \mathcal{N}\left(x ; m_{\gamma, k}^{(i)}, P_{\gamma, k}^{(i)}\right), \\
\beta_{k \mid k-1}(x \mid \zeta)= & \sum_{j=1}^{J_{\beta, k}} w_{\beta, k}^{(j)} \mathcal{N}\left(x ; F_{\beta, k-1}^{(j)} \zeta\right. \\
& \left.+d_{\beta, k-1}^{(j)}, Q_{\beta, k-1}^{(j)}\right)
\end{aligned}
$$

where $w_{\gamma, k}^{(i)}, m_{\gamma, k}^{(i)}, P_{\gamma, k}^{(i)}, J_{\gamma, k}$, are the weights, means, covariances, and a total number of Gaussian components regarding the birth intensity. In the same way $w_{\beta, k}^{(j)}$, $F_{\beta, k-1}^{(j)}, d_{\beta, k-1}^{(j)}, Q_{\beta, k-1}^{(j)}, J_{\beta, k}$ are the weights, means, covariances, and a total number of Gaussian components concerning the spawning intensity of a target with previous state $\zeta$. For more details, see [28].

\section{PHD-BASED SENSOR MANAGEMENT}

Sensor management will be addressed in the Partially Observed Markov Decision Process (POMDP) framework. The elements of a POMDP include an information state represented by the multi-object posterior pdf $\pi_{k}\left(X_{k} \mid Z_{1: k}\right)$, a set of admissible controls $\mathbb{U}_{k}(\cdot)$, and a reward function associated with different control selection $\mathcal{R}(\cdot)$. The problem lies in the fact that at the time when we wish to perform a control action, we have no knowledge of the posterior density that will result from that particular action that has been chosen to be undertaken. In this way, the optimal control action is given by maximizing the expected value of a reward function $\mathcal{R}(\cdot)$ over the set of admissible actions [13], [17], [29]:

$$
u_{k}=\arg \max _{u \in \mathbb{U}_{k}} \mathbb{E}\left[\mathcal{R}\left(u, \pi_{k}\left(X_{k} \mid Z_{1: k}\right), Z_{k+1}(u)\right)\right]
$$

where $u_{k}$ denotes the control vector applied at time $k$; $\mathcal{R}(u, f, Z)$ is the reward function associated with the control $u ; Z_{k+1}(u)$ is the predicted measurement obtained if the control vector $u$ is applied. Reward functions are generally based either on a decrease of uncertainty or an increase of information gain between the predicted and posterior multiobject densities.

\section{A. PENT-based sensor management}

The Posterior Expected Number of Targets (PENT) sensor management was developed by Mahler to solve the problem of the optimal placement of the sensor's field of view (FOV) [14]. PENT selects the control action that maximizes the number of targets to be seen by the sensor. PENT is an objective function constructed using the Predicted Ideal MeasurementSet (PIMS) [5]. By maximizing the value of the PENT reward function, sensor's FOV can be directed to those places where the sensor is most likely to collect the PIMS. When a PHD filter is used, the radar-beam direction based on PENT reward function is given as follows:

$$
u_{k}=\arg \max _{u \in \mathbb{U}_{k}}\left[\int D_{k \mid k}\left(x \mid Z_{k+1}^{P I M S}, u\right)\right]
$$

where $u=\operatorname{atan}\left(x^{(j)} / y^{(j)}\right), \forall j \in\left[1, \cdots, N_{\text {targets }}\right]$ is the sensor control, and $Z_{k+1}^{P I M S}$ is a simulated ideal set of measurements based on PIMS, as proposed by [5], [15].

The PENT sensor management was also extended to consider the tactical significance of a target, resulting in the Predicted Expected Number of Targets of Interest (PENTI) approach [15]. When a PHD filter is applied, the radar-beam direction based on PENTI reward function is given as follows:

$$
u_{k}=\arg \max _{u \in \mathbb{U}_{k}}\left[\int \tau(x) D_{k \mid k}\left(x \mid Z_{k+1}^{P I M S}, u\right)\right]
$$

where $\tau(x) \in[0,1]$ is the tactical importance function for each target [5], [15].

\section{B. ERR-based sensor management}

In this section, we propose to minimize the expected risk reduction in the case of a radar beam-pointing problem where targets need to be prioritized. There is a decision to be made on a target classification. If it is incorrect, it results in a cost, i.e. a loss of a target of interest or a loss of sensor resources. In this paper, an incorrect decision is tagged as being a type 1 error during statistical hypothesis testing. A type 1 error corresponds to an incorrect rejection of a true null hypothesis $H_{0}$, and occurs when it is true yet rejected.

The matrix $C M_{1}$, as defined by Equation 12, contains the cost of committing a type 1 error. Each column represents the true classification, and each row stands for the decision on a classification. The diagonal is zero since there is no cost when the correct decision takes place. 


$$
C M_{1}=\begin{aligned}
& 1 \\
& 2 \\
& \vdots \\
& n
\end{aligned}\left(\begin{array}{cccc}
1 & 2 & \ldots & n \\
0 & c_{1_{12}} & \ldots & c_{1_{1 n}} \\
c_{1_{21}} & 0 & \ldots & c_{1_{2 n}} \\
\vdots & \vdots & \ddots & \vdots \\
c_{1_{n 1}} & c_{1_{n 2}} & \ldots & 0
\end{array}\right)
$$

where each $c_{1_{i j}}$ entry occurs when a decision falsely rejects $H_{0}$, resulting in a type 1 error.

\section{The Expected Cost of Committing a Type 1 Error}

The expected cost of making a type 1 error when deciding on the classification of a target track is influenced by many factors, including the current classification, the probability of the actual target being lost (or not), and the decision about this classification. The above-mentioned factors are modeled by random variables. The expected cost can be obtained by applying the law of total expectation, as described in [22] and detailed below.

Let $C_{1}$ be a discrete random variable representing the cost of the type 1 error. The cost matrix $C M_{1}$ contains entries $\left\{c_{1_{i j}}\right\}$ where each $c_{1_{i j}}$ entry occurs when a decision falsely rejects $H_{0}$, resulting in a type 1 error. $J$ corresponds to a categorical random variable representing the current classification, $\{j \mid j \in[1, n]\}$. $I$ is a categorical random variable denoting the decision on a classification, $\{i \mid i \in[1, n]\}$. $\hat{I}$ is a discrete, uniformly distributed, random variable denoting the classification decision on a reacquired target after it has been lost, $\{i \mid i \in[1, n]\}$. $L$ is a Bernoulli random variable representing whether or not the actual target is lost, where the event space is $\{0,1\}$. We use the law of the iterated expectation for each random variable that determines the expected cost described as:

$$
\begin{aligned}
& E_{c_{1}}\left(C_{1} \mid I=i\right) \\
= & E_{c_{1}}\left(C_{1} \mid I=i, L=1, J=i\right) P(L=1) P(J=i) \\
& +E_{c_{1}}\left(C_{1} \mid I=i, L=1, J \neq i\right) P(L=1) P(J \neq i) \\
& +E_{c_{1}}\left(C_{1} \mid I=i, L=0, J=i\right) P(L=0) P(J=i) \\
& +E_{c_{1}}\left(C_{1} \mid I=i, L=0, J \neq i\right) P(L=0) P(J \neq i) \\
= & E_{c_{1}}\left(C_{1} \mid I=i, L=1, J=i\right) P(L=1) P(J=i) \\
& +E_{c_{1}}\left(C_{1} \mid I=i, L=1, J \neq i\right) P(L=1) P(J \neq i) \\
& +0 \\
& +E_{c_{1}}\left(C_{1} \mid I=i, L=0, J \neq i\right) P(L=0) P(J \neq i)
\end{aligned}
$$

In the above summation, the first term is the expected cost resulting from a situation in which a correct decision is made but the target is lost. The second term represents a wrong decision and a loss of a target. The third term becomes zero since the decision is correct and the target is not lost. As for the fourth term, one can observe that a target is not lost in spite of a wrong decision being made. At this point, it would be necessary to consider the case in which the target would be reacquired. Note that regardless of the classifier's accuracy, it is possible that the acquired target is not the original one. Thus, (13) would be as follows:

$$
\begin{aligned}
& \quad E_{c_{1}}\left(C_{1} \mid I=i\right) \\
& =\quad E_{c_{1}}\left(C_{1} \mid I=i, L=1, J=i, \hat{I}=i\right) P(L=1) P(J=i) P(\hat{I}=i) \\
& +E_{c_{1}}\left(C_{1} \mid I=i, L=1, J=i, \hat{I} \neq i\right) P(L=1) P(J=i) P(\hat{I} \neq i) \\
& +E_{c_{1}}\left(C_{1} \mid I=i, L=1, J \neq i, \hat{I}=i\right) P(L=1) P(J \neq i) P(\hat{I}=i) \\
& +E_{c_{1}}\left(C_{1} \mid I=i, L=1, J \neq i, \hat{I} \neq i\right) P(L=1) P(J \neq i) P(\hat{I} \neq i) \\
& +E_{c_{1}}\left(C_{1} \mid I=i, L=0, J \neq i\right) P(L=0) P(J \neq i) \\
& \quad \\
& \quad+E_{c_{1}}\left(C_{1} \mid I=i, L=1, J=i, \hat{I} \neq i\right) P(L=1) P(J=i) P(\hat{I} \neq i) \\
& +E_{c_{1}}\left(C_{1} \mid I=i, L=1, J \neq i, \hat{I}=i\right) P(L=1) P(J \neq i) P(\hat{I}=i) \\
& +0 \\
& +E_{c_{1}}\left(C_{1} \mid I=i, L=0, J \neq i\right) P(L=0) P(J \neq i)
\end{aligned}
$$

In the above summation, the first term becomes zero once the correct decision has been made, even though the track has been lost and later reacquired. Hence, there is no cost. In the second term, the cost is observed when the target is lost and reacquired and a wrong decision about its classification takes place. In the third term, the cost is present and the target classification decision is never correct, even after the target has been lost and reacquired. The fourth term of summation represents the case in which the classification decision is incorrect (i.e. $I=i$ and $J \neq i$ ), and the reacquired target is characterized by a different classification (i.e. $\hat{I} \neq i$ and $J \neq i$ ). Consequently, the cost is zero because it does not exist with regard to the initial decision of $I=i$ before the track has been lost. The last term illustrates the cost stemming from a wrong target classification since the target is never lost. These terms are related to specific rows and columns of the cost matrix $C M_{1}$ as shown below:

$$
\begin{aligned}
& E_{c_{1}}\left(C_{1} \mid I=i\right) \\
= & E_{c_{1}}\left(C_{1} \mid I=i, L=1, J=i, \hat{I} \neq i\right) P(L=1) P(J=i) P(\hat{I} \neq i) \\
& +E_{c_{1}}\left(C_{1} \mid I=i, L=1, J \neq i, \hat{I}=i\right) P(L=1) P(J \neq i) P(\hat{I}=i) \\
& +E_{c_{1}}\left(C_{1} \mid I=i, L=0, J \neq i\right) P(L=0) P(J \neq i) \\
= & \sum_{c_{1}} c_{1_{i j}}\left(P\left(C_{1}=c_{1_{i j}} \mid I=i, L=1, J=i, \hat{I} \neq i\right)\right. \\
& +\sum_{c_{1}{ }_{i j}} c_{1_{i j}}\left(P\left(C_{1}=c_{1_{i j}} \mid I=i, L=1, J \neq i, \hat{I}=i\right)\right. \\
& +\sum_{c_{1}} c_{1_{i j}} P\left(C_{1}=c_{1_{i j}} \mid I=i, L=0, J \neq i\right) P(L=0) P(J \neq i) \\
= & \sum_{r \in I} c_{1_{r i}} P(L=1) P(J=i) P(\hat{I} \neq i) \\
& +\sum_{r \in J} c_{1_{i r}} P(L=1) P(J=r) P(\hat{I}=i) \\
& +\sum_{r \in J} c_{1_{i r}} P(L=0) P(J=r) \quad \forall r \neq i
\end{aligned}
$$


In Equation 15, the cost of the type 1 error $C_{1}$ for all $r=i$ is zero since there is no cost when the correct decision takes place. Note that the first term in (15) is a function of the rows of the cost matrix over column $J=i$. This implies an incorrect decision after the target was reacquired. Finally, assuming $\hat{I}$ being uniformly distributed, and $P_{\text {lost }}$ the probability of the actual target to be lost, Equation (15) can be rewritten as below:

$$
\begin{aligned}
E_{c_{1}}\left(C_{1} \mid I=i\right) & =\sum_{r \in I} c_{r i} P(J=i) P_{\text {lost }} \frac{n-1}{n} \\
& +\sum_{r \in J} c_{i r} P(J=r) P_{\text {lost }} \frac{1}{n} \\
& +\sum_{r \in J} c_{i r} P(J=r)\left(1-P_{\text {lost }}\right) \quad \forall r \neq i
\end{aligned}
$$

\section{The Expected Risk Reduction}

When a decision on a target classification is made, the goal is to minimize the risk. In this way, the minimum expected cost is chosen among all possible decisions for each track classification. The risk always decreases with new measurements and reduces the probability of the target being misclassified or lost [20]-[23].

The expected risk reduction (ERR) is achieved using the minimum expected cost presented in (16). Note that the probabilities in this equation change as measurements are accumulated by a sensor. It is assumed that this probabilities change as a Bayesian update. Denoting $R$ as the minimum cost before a measurement update, we can calculate the ERR as:

$$
\begin{aligned}
R_{i} & \triangleq E_{c_{1}}\left(C_{1} \mid I=i\right) \\
R & =\min _{i}\left\{R_{i}\right\}
\end{aligned}
$$

Assuming that the posterior probabilities are denoted by $P_{\text {lost }}^{\prime}$ and $P^{\prime}(J=i)$, the risk using these updated probabilities is:

$$
\begin{aligned}
& R^{\prime}=\min _{i}\left\{R_{i}^{\prime}\right\} \\
& =\min _{i}\left\{\begin{array}{c}
\sum_{r \in I} c_{r i} P^{\prime}(J=i) P_{\text {lost }}^{\prime} \frac{n-1}{n} \\
+\sum_{r \in J} c_{i r} P^{\prime}(J=r) P_{\text {lost }}^{\prime} \frac{1}{n} \\
+\sum_{r \in J} c_{i r} P^{\prime}(J=r)\left(1-P_{\text {lost }}^{\prime}\right)
\end{array}\right\}
\end{aligned}
$$

When the classification probability is updated through the direct application of Bayes' theorem, then (19) can be rewritten as follows:

$$
\begin{aligned}
& R^{\prime}=\min _{i}\left\{R_{i}^{\prime}\right\} \\
& =\min _{i}\left\{\begin{array}{c}
\sum_{r \in I} c_{r i} \frac{P(M=m \mid J=i) P(J=i)}{P(M=m)} P_{\text {lost }}^{\prime} \frac{n-1}{n} \\
+\sum_{r \in J} c_{i r} \frac{P(M=m \mid J=r) P(J=r)}{P(M=m)} P_{\text {lost }}^{\prime} \frac{1}{n} \\
+\sum_{r \in J} c_{i r} \frac{P(M=m \mid J=r) P(J=r)}{P(M=m)}\left(1-P_{\text {lost }}^{\prime}\right)
\end{array}\right\}
\end{aligned}
$$

Since any classification measurement $M$ is possible, it is necessary to calculate an additional expectation over all possible measurements $\left\langle R^{\prime}\right\rangle$ which can be illustrated as:

$$
\begin{aligned}
\left\langle R^{\prime}\right\rangle= & \sum_{m \in M} R^{\prime} P(M=m) \\
= & \sum_{m \in M} \min _{i}\left\{\begin{array}{c}
\sum_{r \in I} c_{r i}(P(M=m \mid J=i) P(J=i) \\
\left.P_{\text {lost }}^{\prime} \frac{n-1}{n}\right) \\
+\sum_{r \in J} c_{i r}(P(M=m \mid J=r) P(J=r) \\
\left.P_{\text {lost }}^{\prime} \frac{1}{n}\right) \\
+\sum_{r \in J} c_{i r}(P(M=m \mid J=r) P(J=r) \\
\left.\left(1-P_{\text {lost }}^{\prime}\right)\right)
\end{array}\right\}
\end{aligned}
$$

Taking into consideration that the expected cost decreases in value with new measures [20], [22], [23], the radar-beam direction based on ERR is given as follows:

$$
u_{k}=\arg \max _{u \in \mathbb{U}_{k}}[\operatorname{ERR}(u)]
$$

where $u=\operatorname{atan}\left(x^{(j)} / y^{(j)}\right), \forall j \in\left[1, \cdots, N_{\text {targets }}\right]$ is the sensor control, and $\operatorname{ERR}(u)=R^{(j)}-\left\langle R^{\prime(j)}\right\rangle$. The sensor control that provides the greatest reduction in this ERR value is chosen to take the actual measurement.

\section{NuMERICAL EXAMPLE}

In order to demonstrate the proposed approach we use a numerical example where a multifunction radar (MFR) is controlled to track an unknown number of targets. It is able to track targets in the sector defined by $[0,2000]$ meters in range and $[-\pi / 2, \pi / 2]$ rad in bearing using its "pencil" beam. The radar has a 4-degree beamwidth. The true trajectories are shown in Figure 1. The duration of the scenario is 300 seconds.

For each target, there is information about kinematic true state and its classification. Both data are represented by $X$ as follows:

$$
X=\left[X_{\text {kinematic }} \quad X_{\text {classification }}\right]
$$

A nearly constant turn kinematics model having a varying turn rate is considered [30]. Thus, the true kinematic state 


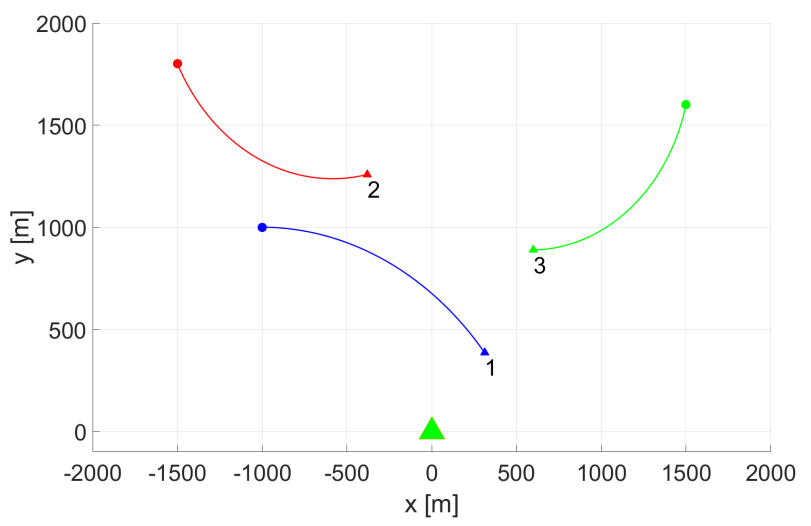

Fig. 1. The trajectories of three observed targets. The radar is at the origin of the axes. Start and stop positions for each track are shown with $\circ$ and $\triangle$.

consists of a two dimensional position, velocity and the turn rate:

$$
X_{\text {kinematic }}=[x, \dot{x}, y, \dot{y}, \omega]^{T}
$$

The transition model in target tracking can be formulated as follows:

$$
\begin{aligned}
x_{k} & =F\left(\omega_{k-1}\right) x_{k-1}+G w_{k-1} \\
\omega_{k} & =\omega_{k-1}+T u_{k-1}
\end{aligned}
$$

where

$$
F(\omega)=\left[\begin{array}{cccc}
1 & \frac{\sin \omega T}{\omega} \omega & 0 & -\frac{1-\cos \omega T}{\omega} \\
0 & \cos \omega T & 0 & -\sin \omega T \\
0 & \frac{1-\cos \omega T}{\omega} \omega & 1 & \frac{\sin \omega T}{\cos \omega T} \\
0 & \sin \omega T & 0 & \cos \omega T
\end{array}\right], G=\left[\begin{array}{cc}
\frac{T^{2}}{2} & 0 \\
T & 0 \\
0 & \frac{T^{2}}{2} \\
0 & T
\end{array}\right],
$$

$T=1 s$ is the sampling period; $w_{k-1} \sim \mathcal{N}\left(\cdot ; 0, \sigma_{w}^{2} I\right)$ and $u_{k-1} \sim \mathcal{N}\left(\cdot ; 0, \sigma_{u}^{2} I\right)$ are the process noise with standard deviation $\sigma_{w}=15 \mathrm{~m} / \mathrm{s}^{2}$ and $\sigma_{u}=(\pi / 180) \mathrm{rad} / \mathrm{s}$. The targets are observed via a radar that provides range and bearing measurements. Each target is detected with probability $p_{D, k}=0.98$, and the measurement uses the observation model given by:

$$
z_{k}=\left[\begin{array}{c}
\arctan (x / y) \\
\sqrt{x^{2}+y^{2}}
\end{array}\right]+\varepsilon_{k}
$$

where $\varepsilon_{k} \sim \mathcal{N}\left(\cdot ; 0, R_{k}\right) ; R_{k}=\operatorname{diag}\left(\left[\sigma_{\theta}^{2}, \sigma_{r}^{2}\right]\right)$ is the measurement noise covariance matrix, with $\sigma_{\theta}=(0.5 \pi / 180) \mathrm{rad}$, and $\sigma_{r}=10 \mathrm{~m}$. The detected measurements are immersed in clutter which is typically modeled as a Poisson RFS with intensity function:

$$
\kappa_{k}(z)=\lambda_{c} V u(z)
$$

where $u(\cdot)$ represents the uniform density over the surveillance region, $V=3.14 \times 10^{5}(\mathrm{radm})$ is the area of the surveillance region, and $\lambda_{c}=3.18 \times 10^{-5}(\mathrm{radm})^{-1}$ is the average clutter intensity. In that case, an average of 10 clutter points per scan is received with $P_{F A}=0.10$ at each time instance.

The classification state estimate is formulated hereafter. Assuming that there are $n$ possible classification states for each target, $J$ is a random variable that stands for the true classification with support $\{j \mid j \in[1, n]\}$.

$$
X_{\text {classification }}=\left[\begin{array}{c}
P(J=1) \\
\vdots \\
P(J=n)
\end{array}\right]
$$

The classification probability is updated by applying the Bayes' theorem as shown below. The classification measurement is represented by a discrete random variable $M$ with support $\{m \mid m \in[1, n]\}$.

$$
\begin{aligned}
P^{\prime}(J=i) & \triangleq P(J=i \mid M=m) \\
& =\frac{P(M=m \mid J=i) P(J=i)}{P(M=m)} \\
& =\frac{P(M=m \mid J=i) P(J=i)}{\sum_{r=1}^{n} P(M=m \mid J=r) P(J=r)}
\end{aligned}
$$

where $P^{\prime}$ indicates the posterior probability. To simplify classification notations in this paper, measurement likelihoods $P(M=m \mid J=i)$ are represented by a normalized confusion matrix $C C$.

$$
C C=\vdots \begin{array}{ccc}
1 & \ldots & n \\
n
\end{array}\left(\begin{array}{ccc}
P(M=1 \mid J=1) & \ldots & P(M=1 \mid J=n) \\
\vdots & \ddots & \vdots \\
P(M=n \mid J=1) & \ldots & P(M=n \mid J=n)
\end{array}\right)
$$

The radar has a 4-degree beamwidth. If the target ground truth corresponding to the track position is outside this FOV (i.e. the state estimate is very poor), the track is considered lost. The probability $P_{\text {lost }}$ is assumed to be the portion of a multivariate normal distribution $\mathcal{N}\left(\hat{x}_{k}, \hat{P}_{k}\right)$ not contained in the sensor's FOV when the sensor's aim-point is centered on a kinematic state of the target ( $\hat{x}$ is the mean state estimate and $\hat{P}$ is the state estimate covariance).

In this paper we use the GM-PHD tracker to propagate a parametrized approximation to the multi-target posterior applying the gating and pruning/merging procedures. Gating is performed at each time step using a $99 \%$ validation gate (the region centered on the predicted measurement with a 0.99 probability of containing a primary object generated measurement), as described in [3], [4]. Pruning and merging are performed at each time step using a weight threshold of $T_{t h}=10^{-5}$ and a merging threshold of $U=4 \mathrm{~m}$, see [25].

For comparison purposes, the Expected Risk Reduction (ERR) approach was contrasted with three different sensor management methods involving the Posterior Expected Number of Targets (PENT), the Posterior Expected Number of 
Targets of Interest (PENTI), and the random assignment. One thousand Monte Carlo runs were conducted using each method. All target classifications are initially unknown and each target starts with a high accuracy kinematic track. Therefore, the main tasks of the radar are to correctly classify, track the targets and allocate measurements to the target of interest. Tracking accuracy is measured using the Optimal Sub-Pattern Assignment (OSPA) metric [31]. The OSPA measures the error between the true and the estimated multi-target states, $X_{k}$ and $\hat{X}_{k}$, respectively. Two simulation examples are used to test the proposed approach to sensor management. An additional example can be found in [23].

1) Example 1: In order to evaluate the expected risk reduction (ERR) metric, a binary classification state is considered where the target to be tracked is either a target of interest $(J=1)$ or a target of non interest $(J=2)$. The binary classification measurement $M$ has support $\{m \mid m \in[1,2]\}$. Cost matrix $C M_{1}$ and confusion matrix $C C$ are:

$$
C M_{1}=\frac{1}{2}\left(\begin{array}{cc}
1 & 2 \\
0 & 1 \\
30 & 0
\end{array}\right) \quad C C=\begin{gathered}
1 \\
2 \\
2
\end{gathered}\left(\begin{array}{cc}
0.8 & 0.2 \\
0.2 & 0.8
\end{array}\right)
$$

In this example, targets 2 and 3 are those of interest and, consequently, should be prioritized. Figure 2 shows a typical run of the proposed algorithm at the instant $k=40$ seconds.

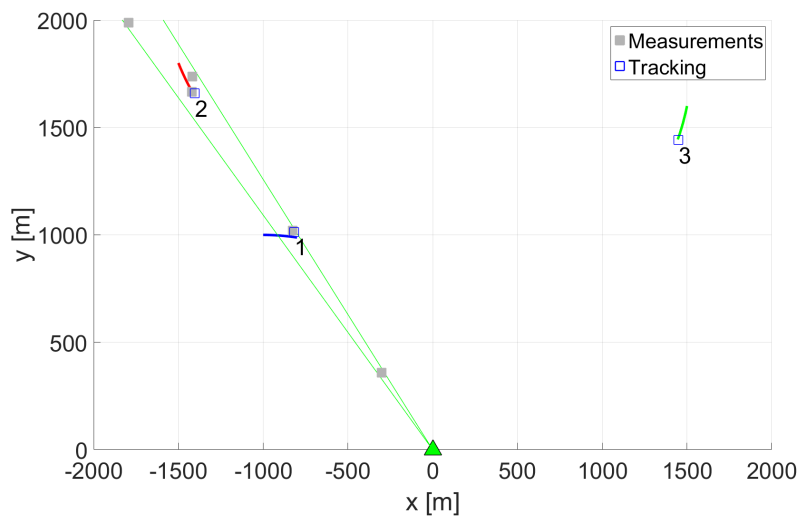

Fig. 2. Typical run to the ERR approach at instant $k=40$ seconds. The radar is at the origin of the axes. Target tracking and identification are provided using the GM-PHD tracker.

The ERR was compared with the PENTI and the random sensor management. Of all three sensor management approaches, the OSPA metric, along with the cardinality estimation and localization error, is averaged over 1000 independent Monte Carlo runs. In Figure 3, the Monte Carlo average of the OSPA distance for $p=1$ and $c=100 \mathrm{~m}$ is shown. It can be observed that the curves stabilize to an average error close to $60 \mathrm{~m}$ per target for the PENTI and the random schemes, and close to $50 \mathrm{~m}$ per target for the ERR approach.

Examining the cardinality estimation and the localization error given in Figures 4 and 5, it can be seen that in terms of localization error, the PENTI and ERR sensor management

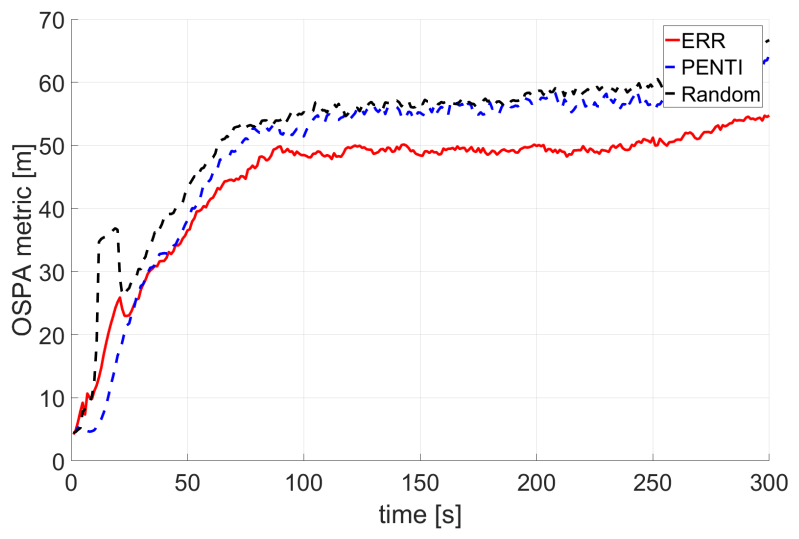

Fig. 3. OSPA metric. Error performance of the three sensor management approaches with target prioritization, averaged over 1000 Monte Carlo runs.

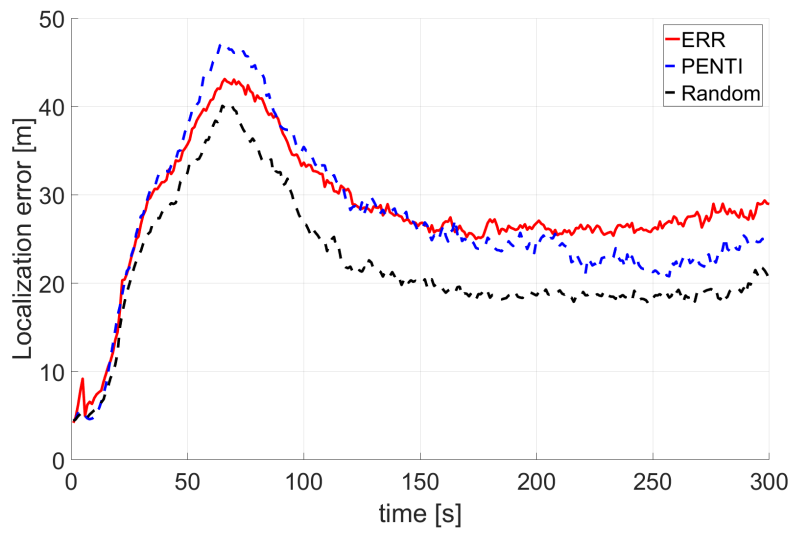

Fig. 4. Localization error performance of the three sensor management approaches with target prioritization, averaged over 1000 Monte Carlo runs.

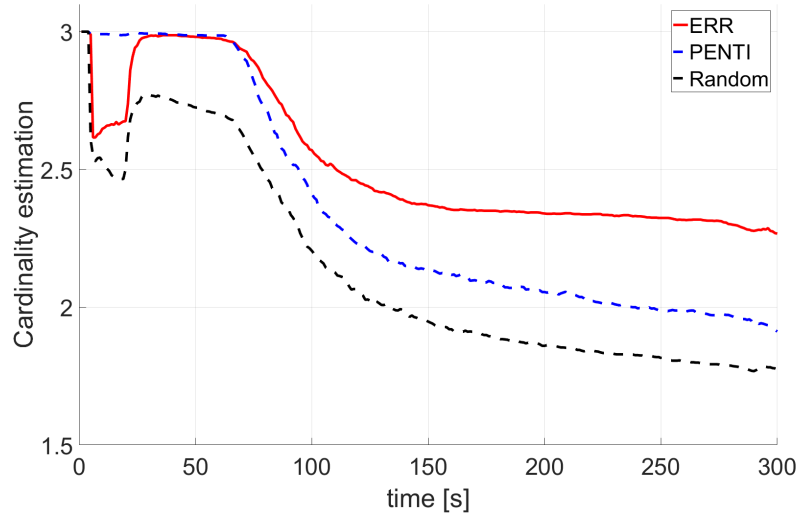

Fig. 5. Estimated number of targets for three sensor management approaches with target prioritization, averaged over 1000 Monte Carlo runs.

settle to an error consistent with the standard deviation of the measurement noise, while the random schedule achieves an unexpectedly lower localization error. Analyzing Figure 5, we can observe that, on average, the random method can keep 
track only on 2 targets, while the ERR method is capable of tracking about 2.5 targets. The ERR approach is penalized much more than the PENTI and the random scheme since it is more successful at keeping track on more targets at the expense of the localization estimation which is less accurate. In fact, in terms of cardinality estimation, the ERR scheme is at a significant advantage in this scenario.

2) Example 2: In order to evaluate the ERR metric without prioritization, a mono classification state is considered. In that case, each target belongs to precisely the same class $(J=1)$, and targets 1, 2, and 3 should be tracked with the same priority. In this example, the ERR is compared with the PENT and the random sensor management. The OSPA metric, as well as the cardinality estimation and localization error of all three sensor management approaches are averaged over 1000 independent Monte Carlo runs.

Figure 6 shows the Monte Carlo average of the OSPA distance for $p=1$ and $c=100 \mathrm{~m}$. The ERR sensor management presents a behavior quite similar to the PENT approach. It can be seen that after the initial settle-in phase the curves stabilize to an average error close to $50 \mathrm{~m}$ per target for the PENT and the ERR approaches, and close to $60 \mathrm{~m}$ per target for the random schemes. Examining the cardinality estimation and the localization error given in Figures 7 and 8 , it can be noticed that the random schedule achieves an unexpectedly lower localization error, with a similar result to example 1.

As far as the localization error is concerned, the results indicate that the ERR and the PENT approaches are penalized much more than the random scheme since they are more successful at keeping track on more targets at the expense of the localization estimation which is less accurate. Even though the PENT and the PENTI approaches turned out to be effective in this case study, they are not expected to perform well when confronted with target localization problems [16], [18]. To get a deeper insight into pros and cons of each method, the performance analyses regarding other scenarios are required.

\section{Conclusion}

This paper introduces the Expected Risk Reduction (ERR) approach to sensor management in the case of a radar beampointing problem by combining POMDP theory and RFS framework. The GM-PHD tracker was employed to efficiently but approximately propagate the multi-target posterior density, which was then used to calculate a reward function in order to determine the sensor control. The ERR is based on the expected cost of an incorrect decision on target's classification. This cost was then conditioned on the event of losing a target track which allowed for achieving the combination of a classification and kinematic uncertainty in the same metric. It has been put forward that the ERR approach can maintain a track on targets of interest when it is not possible for a single sensor to track all targets in the environment. The numerical example demonstrated the effectiveness of the proposed reward function for PHD filtering in a radar beampointing problem where targets need to be prioritized. Future works will investigate computationally efficient solutions for PHD and Multi-Bernoulli filtering with multiple-steps-ahead sensor control as well as sensor management in distributed fusion architecture.

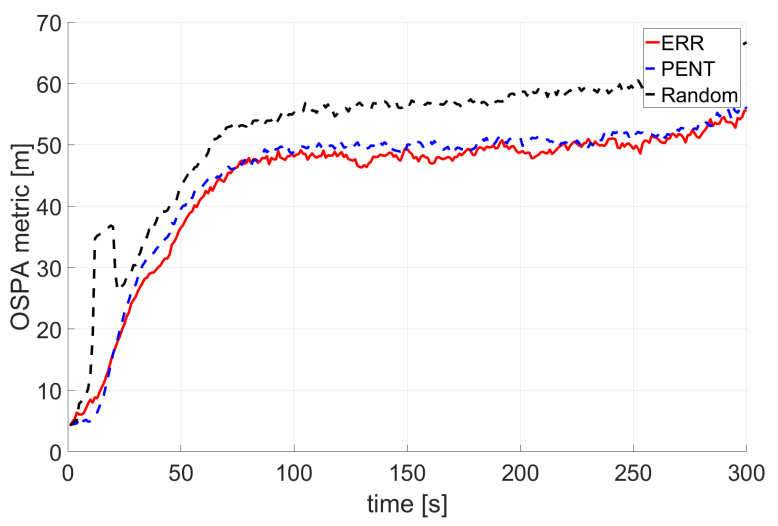

Fig. 6. OSPA metric. Error performance of the three sensor management approaches without target prioritization, averaged over 1000 Monte Carlo runs.

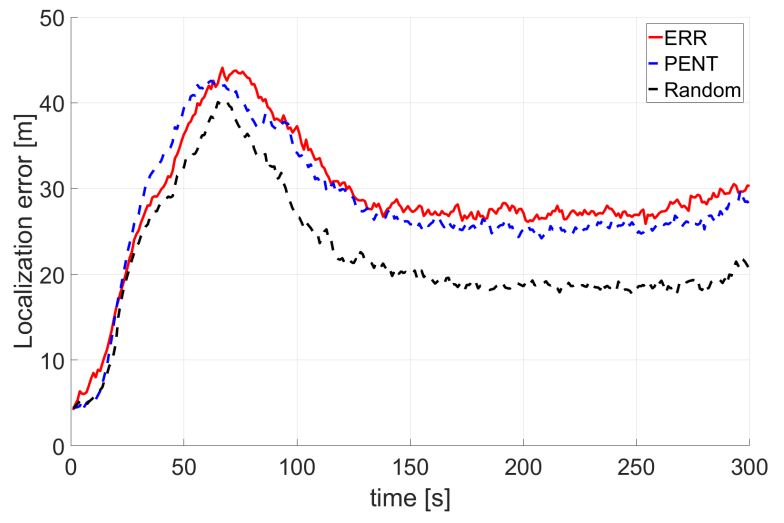

Fig. 7. Localization error performance of the three sensor management approaches without target prioritization, averaged over 1000 Monte Carlo runs.

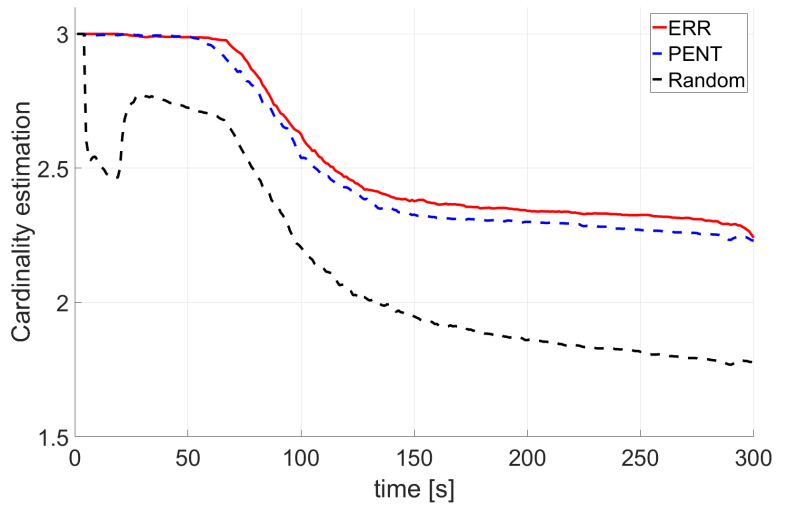

Fig. 8. Estimated number of targets for three sensor management approaches without target prioritization, averaged over 1000 Monte Carlo runs. 


\section{ACKNOWLEDGMENT}

The authors would like to thank the CNPq (Brazilian agency) and ANRT for their support.

\section{REFERENCES}

[1] A. O. Hero III, D. A. Castañón, D. Cochran, and K. Kastella, Foundations and Applications of Sensor Management. USA: Springer Science \& Business Media, 2008.

[2] A. O. Hero III and D. Cochran, "Sensor Management: Past, Present, and Future," IEEE Sensors Journal, vol. 11, no. 12, pp. 3064-3075, Dec 2011.

[3] S. Blackman and R. Popoli, Design and Analysis of Modern Tracking Systems. Norwood, MA: Artech House, 1999.

[4] Y. Bar-Shalom, P. K. Willet, and X. Tian, Tracking and Data Fusion: A Handbook of Algorithms. YBS Publishing, 2011.

[5] R. P. S. Mahler, Advances in statistical Multisource-Multitarget Information Fusion. Artech House, 2014.

[6] —, "Multitarget Bayes Filtering via First-Order Multitarget Moments," IEEE Transactions on Aerospace and Electronic Systems, vol. 39, no. 4, pp. 1152-1178, Oct 2003.

[7] B.-N. Vo, S. Singh, and A. Doucet, "Sequential Monte Carlo methods for Multi-target Filtering with Random Finite Sets," IEEE Transactions on Aerospace and Electronic Systems, vol. 41, no. 4, pp. 1224-1245, Oct 2005.

[8] R. P. S. Mahler, "PHD Filters of Higher Order in Target Number," IEEE Transactions on Aerospace and Electronic Systems, vol. 43, no. 4, pp 1523-1543, Oct 2007.

[9] - Statistical Multisource Multitarget Information Fusion. Artech House, 2007.

[10] B.-T. Vo, B.-N. Vo, and A. Cantoni, "The Cardinality Balanced MultiTarget Multi-Bernoulli Filter and its Implementations," IEEE Transactions on Signal Processing, vol. 57, no. 2, pp. 409-423, Feb 2009.

[11] B.-T. Vo and B.-N. Vo, "Labeled random finite sets and multi-object conjugate priors," IEEE Transactions on Signal Processing, vol. 61, no. 13 , pp. $3460-3475,2013$.

[12] B.-N. Vo, B.-T. Vo, and D. Phung, "Labeled random finite sets and the Bayes multi-target tracking filter,' IEEE Transactions on Signal Processing, vol. 62, no. 24, pp. 6554-6567, 2014.

[13] R. P. S. Mahler, "Objective functions for Bayesian control-theoretic sensor management, 1: Multitarget first-moment approximation," in Aerospace Conference, 2003. Proceedings. 2003 IEEE, vol. 4. IEEE, March 2003, pp. 4_1905-4_1923.

[14] R. P. S. Mahler and T. R. Zajic, "Probabilistic objective functions for sensor management," Proc. SPIE, vol. 5429, pp. 233-244, 2004.

[15] R. Mahler, "Multitarget sensor management of dispersed mobile sensors," in Theory and algorithms for cooperative systems. World Scientific, 2004, pp. 239-310.

[16] E. Delande, "Multi-sensor PHD Filtering with Application to Sensor Management," Ph.D. dissertation, Ecole Centrale de Lille, Laboratoire d'Automatique, Génie Informatique et Signal, Lille, France, 2012.

[17] B. Ristic and B.-N. Vo, "Sensor control for multi-object state-space estimation using random finite sets," Automatica, vol. 46, no. 11, pp. 1812-1818, 2010.

[18] B. Ristic, B.-N. Vo, and D. Clark, "A note on the reward function for PHD filters with sensor control," IEEE Transactions on Aerospace and Electronic Systems, vol. 47, no. 2, pp. 1521-1529, 2011.

[19] H. G. Hoang, B. Vo, B. Vo, and R. P. S. Mahler, "The Cauchy-Schwarz divergence for Poisson point processes," CoRR, vol. abs/1312.6224, 2013.

[20] D. Papageorgiou and M. Raykin, "A risk-based approach to sensor resource management," in Advances in Cooperative Control and $\mathrm{Op}$ timization. Springer, 2007, pp. 129-144.

[21] Y. Wang, I. I. Hussein, and R. S. Erwin, "Risk-Based Sensor Management for Integrated Detection and Estimation," in American Control Conference, June 2011, pp. 3633-3638.

[22] S. R. Martin, "Risk-based Sensor Resource Management for Field of View Constrained Sensors," in 18th International Conference on Information Fusion (Fusion), 2015, July 2015, pp. 2041-2048.

[23] M. E. Gomes-Borges, D. Maltese, P. Vanheeghe, G. Sella, and E. Duflos, "Sensor Management using Expected Risk Reduction approach," in Information Fusion (Fusion), 2016 19th International Conference on. IEEE, 2016, pp. 2050-2058.
[24] D. E. Clark, K. Panta, and B.-N. Vo, "The GM-PHD Filter Multiple Target Tracker," in 9th International Conference on Information Fusion, Jul 2006, pp. 1-8.

[25] K. Panta, B.-N. Vo, and D. E. Clark, "An Efficient Track Management Scheme for the Gaussian-Mixture Probability Hypothesis Density Tracker," in 4th International Conference on Intelligent Sensing and Information Processing. ICISIP 2006., Oct 2006, pp. 230-235.

[26] D. Maltese and A. Lucas, "Data fusion: Quite Silent Search Function in Naval Air Defense," in Proceedings of SPIE AeroSense, vol. 3698, 1999, pp. 36-47.

[27] B.-N. Vo and W.-K. Ma, "A closed-form solution for the Probability Hypothesis Density filter," in 7th International Conference on Information Fusion, vol. 2, Jul 2005, p. 8 pp.

[28] _ - "The Gaussian Mixture Probability Hypothesis Density Filter," IEEE Transactions on Signal Processing, vol. 54, no. 11, pp. 40914104, Nov 2006.

[29] M. Beard, B.-T. Vo, B.-N. Vo, and S. Arulampalam, "Sensor control for multi-target tracking using cauchy-schwarz divergence," in Information Fusion (Fusion), 2015 18th International Conference on. IEEE, 2015, pp. 937-944.

[30] X. R. Li and V. P. Jilkov, "Survey of Maneuvering Target Tracking. Part I: Dynamic Models," IEEE Transactions on Aerospace and Electronic Systems, vol. 39, no. 4, pp. 1333-1364, Oct 2003.

[31] D. Schuhmacher, B.-T. Vo, and B.-N. Vo, "A Consistent Metric for Performance Evaluation of Multi-Object Filters," IEEE Transactions on Signal Processing, vol. 56, no. 8, pp. 3447-3457, 2008. 